\title{
New far-field extrapolation method for the computation of electric fields
}

\author{
XIN YANG \\ College of Big Data and Information Engineering, Guizhou University, \\ Guiyang 550025, China
}

Semiconductor Power Device Reliability Engineering Research Center of Ministry of Education, Guiyang 550025, China

Key Laboratory of Micro-Nano-Electronics and Software Technology of Guizhou Province, Guiyang 550025, China

\begin{abstract}
The extrapolation of the electric field is studied theoretically both in frequency domain and time domain. Combining Gauss's law with the approximation method in engineering, two new formulas for the scattering field calculation are derived from different logical ideas based on Stratton-Chu formula. The consistency property of the derived formulas is investigated, and the third formula for the scattering field calculation is further obtained. Finally, the time-domain extrapolation is discussed based on the formulas, followed by a simple numerical example. The results obtained are characterized by a simple form and intuitive physical meaning, and are helpful to calculate certain engineering problems.
\end{abstract}

Keywords: far-field extrapolation, Stratton-Chu formula, Gauss's law.

\section{Introduction}

Many researches on the field extrapolation have been conducted by using numerical methods [1- $\underline{5}]$. However, theoretical research on this topic is relatively rare, except StRATtON and ChU who derived the famous equations named after their names $[\underline{6}, \underline{7}]$, i.e., the Stratton-Chu formula. Figure 1 presents the schematic diagram of the Stratton -Chu formula to calculate the far field from a scattering object, in which the far electric field is calculated from:

$$
\left\{\begin{array}{l}
\mathbf{E}_{\mathrm{s}}(\mathbf{r}, \omega)=\oiint_{S}\left\{\begin{array}{l}
j \omega \mu\left[\hat{\mathbf{n}} \times \mathbf{H}\left(\mathbf{r}^{\prime}, \omega\right)\right] G+\left[\hat{\mathbf{n}} \times \mathbf{E}\left(\mathbf{r}^{\prime}, \omega\right)\right] \times \nabla^{\prime} G \\
+\left[\hat{\mathbf{n}} \cdot \mathbf{E}\left(\mathbf{r}^{\prime}, \omega\right)\right] \nabla^{\prime} G
\end{array}\right\} \mathrm{d} s^{\prime} \\
Z_{0}=\sqrt{\frac{\mu}{\varepsilon}}, \quad G\left(\mathbf{r}, \mathbf{r}^{\prime}\right)=\frac{\exp \left(j k\left|\mathbf{r}-\mathbf{r}^{\prime}\right|\right)}{4 \pi\left|\mathbf{r}-\mathbf{r}^{\prime}\right|}
\end{array}\right.
$$




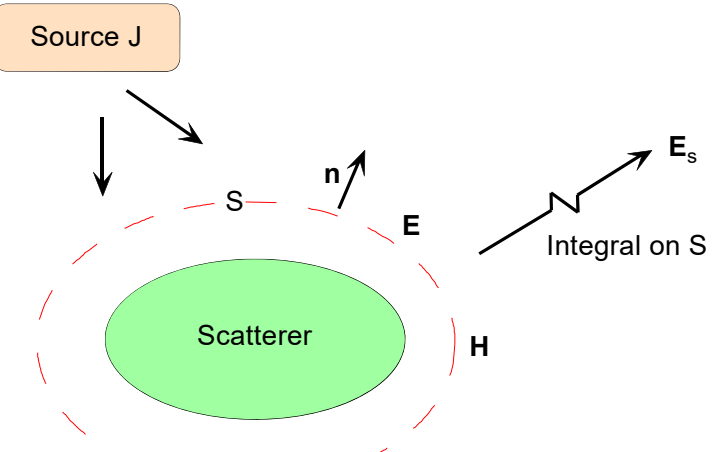

Fig. 1. Schematic of Stratton-Chu formula to calculate the far field.

where $S$ is a closed curved surface surrounding the scatterer; $\mathbf{E}$ and $\mathbf{H}$ represent the electromagnetic fields on the curved surface. Equation (1) is the general formula for the calculation of the scattering field, which thus seems too complicated when dealing with specific computation, such as the vector operations and the closed surface integration. Obviously, such formula form may be not convenient for engineering applications. To solve this problem, the physical optics (PO) method was proposed based on the Stratton-Chu formula [ $\underline{8}-10]$. This method requires integral calculation based on the surface of the object and is mainly applied to metal targets, which therefore limits the universality of the method. In terms of current research status, it is still necessary to further study the scattering principle so as to develop a more concise and versatile scattering analysis method.

According to Gauss's law, the integral of Eq. (1) over the closed surface $S$ is transformed into an integral over an infinite plane. Considering the fact that engineering calculations allow certain calculation errors, so the infinite plane integral becomes an integral over a finite plane by using approximation methods. The significance of this integral transformation is that the closed-surface integral form can be converted into a finite plane integral, which greatly reduces the computational complexity. Then, two new expressions are derived from Eq. (1) by using different methods; the consistency property between the expressions is discussed. Finally, the scattering field computation in time domain is discussed as well. The results obtained are characterized by simple form and intuitive physical meaning. The consistency property and numerical example confirm the correctness of the results in the paper.

\section{Method}

\subsection{Integral domain transformation}

The first step is to transform the integral domain of Eq. (1), as shown in Fig. 2. Overall, Fig. 2a contains three surfaces: (1) a closed curved surface $S$ surrounding the scatterer; 


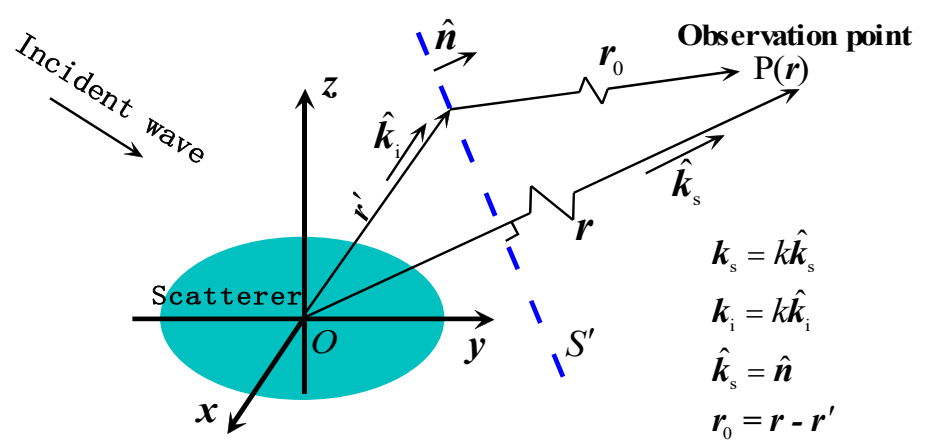

b

Fig. 2. Integral domain transformation and parameter setting. (a) Integral domain transformation, and (b) scattering parameter setting.

(2) an infinite plane $S^{\prime \prime}$; and (3) a finite plane $S^{\prime}$, which is a part of the infinite plane $S^{\prime \prime}$. When calculating the far scattering fields $\mathbf{E}_{\mathrm{s}}$, an appropriate selection of the three surfaces can simplify the integral of Eq. (1) into an integral over the finite plane $S^{\prime}$; and thus new equations to calculate the scattering fields result. The simplification process is presented in detail below, and all parameters are described in Cartesian coordinate system.

As shown in Fig. 2, the infinite plane $S^{\prime \prime}$ is located at the outside of the closed curved surface $S$, whose normal unit vector $\hat{\mathbf{n}}$ has the same direction as the unit vector $\hat{\mathbf{k}}_{\mathrm{s}}$, in the scattering observation direction, i.e., $\hat{\mathbf{n}}=\hat{\mathbf{k}}_{\mathrm{s}}$. It can be inferred from Gauss's law that the contribution of the scattering fields through the closed surface $S$ to the observation point $P(\mathbf{r})$ is equivalent to that through the infinite plane $S^{\prime \prime}$. Therefore, when calculating the scattering fields at the observation point $P(\mathbf{r})$, the integral of Eq. (1) over the closed surface $S$ can be equivalent to the integral over the infinite plane $S^{\prime \prime}$. Theoretically speaking, the scattering fields from scatterer can be full of entire $S^{\prime \prime}$. 
However, considering from the engineering point of view, the main scattering contribution to the observation point $P(\mathbf{r})$ comes from the finite surface $S^{\prime}$. Thus, the integral of Eq. (1) over the infinite plane $S^{\prime \prime}$ is actually approximated to the integral over the finite plane $S^{\prime}$. It is obvious that $S^{\prime}$ is related to the requirement of the calculation accuracy. Based on this idea, the Eq. (1) can be further simplified.

To facilitate the subsequent discussion, the result of the Green's function $G$ from the operators $\nabla$ and $\nabla^{\prime}$ in Eq. (1) should be first determined, where they represent the spatial differential operator at the scattering observation point and the electromagnetic source point, respectively. As shown in Fig. $2 \mathbf{b}$, when a plane wave is incident on the scatterer, there are:

$$
\nabla G=\nabla\left(\frac{\exp \left(j k\left|\mathbf{r}_{0}\right|\right)}{4 \pi\left|\mathbf{r}_{0}\right|}\right)=\nabla\left(\frac{\exp \left(j\left(k r-\mathbf{k}_{\mathrm{s}} \cdot \mathbf{r}^{\prime}\right)\right)}{4 \pi\left(r-\hat{\mathbf{k}}_{\mathrm{s}} \cdot \mathbf{r}^{\prime}\right)}\right)
$$

Considering the scattering far field, the following relationship exists:

$$
r \gg \hat{\mathbf{k}}_{\mathrm{s}} \cdot \mathbf{r}^{\prime}
$$

Then, Eq. (2) can be simplified as:

$$
\begin{aligned}
\nabla G & \approx \nabla\left(\frac{\exp \left(j\left(k r-\mathbf{k}_{\mathrm{s}} \cdot \mathbf{r}^{\prime}\right)\right)}{4 \pi r}\right) \\
& =\frac{1}{4 \pi r} \nabla \exp \left(j\left(k r-\mathbf{k}_{\mathrm{s}} \cdot \mathbf{r}^{\prime}\right)\right)+\exp \left(j\left(k r-\mathbf{k}_{\mathrm{s}} \cdot \mathbf{r}^{\prime}\right)\right) \nabla \frac{1}{4 \pi r} \\
& =j \mathbf{k}_{\mathrm{s}} \frac{\exp \left(j\left(k r-\mathbf{k}_{\mathrm{s}} \cdot \mathbf{r}^{\prime}\right)\right)}{4 \pi r}-\frac{\exp \left(j\left(k r-\mathbf{k}_{\mathrm{s}} \cdot \mathbf{r}^{\prime}\right)\right)}{4 \pi r^{2}} \hat{\mathbf{k}}_{\mathrm{s}}
\end{aligned}
$$

where $r^{2}$ and $r$ correspond to the Coulomb field and the scattering field, respectively. When considering merely the far-field scattering, the Coulomb field in Eq. (3) can be ignored. So the first-order approximation of Eq. (3) is obtained:

$$
\nabla G \approx j \mathbf{k}_{\mathrm{s}} G
$$

It should be noted that, the incident wave vector $\mathbf{k}_{\mathrm{i}}$ is determined by the incident field at the point $\mathbf{r}^{\prime}$, since the scattering calculation is based on the electromagnetic fields on the plane $S^{\prime}$. Finally, according to $r_{0}=\left|\mathbf{r}-\mathbf{r}^{\prime}\right|$, the following relation results:

$$
\nabla^{\prime} G=-j \mathbf{k}_{\mathrm{s}} G
$$

Substituting Eqs. (3) and (4) into Eq. (1) and using appropriate mathematical methods, a more concise formula to calculate the scattering fields can be derived. 


\subsection{Derivation of the new formulas}

\subsubsection{Equation of the first kind}

Firstly, preliminary simplification is given to Eq. (1) based on the closed surface $S$ in Fig. 2. Substituting Eqs. (3) and (4) into Eq. (1), we can obtain:

$$
\begin{aligned}
\mathbf{E}_{\mathrm{s}}(\mathbf{r}, \omega)=\int_{S}\left\{\begin{array}{l}
j \omega \mu\left[\hat{\mathbf{n}} \times \mathbf{H}\left(\mathbf{r}^{\prime}, \omega\right)\right] G \\
+\left[\hat{\mathbf{n}} \times \mathbf{E}\left(\mathbf{r}^{\prime}, \omega\right)\right] \times \nabla^{\prime} G \\
+\left[\hat{\mathbf{n}} \cdot \mathbf{E}\left(\mathbf{r}^{\prime}, \omega\right)\right] \nabla^{\prime} G
\end{array}\right\} \mathrm{d} s^{\prime} \\
=\int_{S} G\left\{\begin{array}{l}
j \omega \mu\left[\hat{\mathbf{n}} \times \mathbf{H}\left(\mathbf{r}^{\prime}, \omega\right)\right] \\
\left.+\left[\hat{\mathbf{n}} \times \mathbf{E}\left(\mathbf{r}^{\prime}, \omega\right)\right] \times\left(-j \mathbf{k}_{\mathrm{s}}\right)\right\} \mathrm{d} s^{\prime} \\
+\left[\hat{\mathbf{n}} \cdot \mathbf{E}\left(\mathbf{r}^{\prime}, \omega\right)\right]\left(-j \mathbf{k}_{\mathrm{s}}\right)
\end{array}\right] \\
=j \frac{\exp (j k r)}{4 \pi r} \oiiint_{S}\left\{\begin{array}{l}
\omega \mu\left[\hat{\mathbf{n}} \times \mathbf{H}\left(\mathbf{r}^{\prime}, \omega\right)\right] \\
-\left[\hat{\mathbf{n}} \times \mathbf{E}\left(\mathbf{r}^{\prime}, \omega\right)\right] \times \mathbf{k}_{\mathrm{s}} \\
-\left[\hat{\mathbf{n}} \cdot \mathbf{E}\left(\mathbf{r}^{\prime}, \omega\right)\right] \mathbf{k}_{\mathrm{s}}
\end{array}\right\} \exp \left(-j \mathbf{k}_{\mathrm{s}} \cdot \mathbf{r}^{\prime}\right) \mathrm{d} s^{\prime} \\
=j k \frac{\exp (j k r)}{4 \pi r} \oiint_{S}\left\{\begin{array}{l}
Z_{0}\left[\hat{\mathbf{n}} \times \mathbf{H}\left(\mathbf{r}^{\prime}, \omega\right)\right] \\
-\left[\hat{\mathbf{n}} \times \mathbf{E}\left(\mathbf{r}^{\prime}, \omega\right)\right] \times \hat{\mathbf{k}}_{\mathrm{s}} \\
-\left[\hat{\mathbf{n}} \cdot \mathbf{E}\left(\mathbf{r}^{\prime}, \omega\right)\right] \hat{\mathbf{k}}_{\mathrm{s}}
\end{array}\right\} \exp \left(-j \mathbf{k}_{\mathrm{s}} \cdot \mathbf{r}^{\prime}\right) \mathrm{d} s^{\prime}
\end{aligned}
$$

Considering the plane incident wave in free space, the relationship between electric field and magnetic field is determined by:

$$
\mathbf{H}\left(\mathbf{r}^{\prime}, \omega\right)=\frac{1}{Z_{0}} \hat{\mathbf{k}}_{\mathrm{i}}\left(\mathbf{r}^{\prime}\right) \times \mathbf{E}\left(\mathbf{r}^{\prime}, \omega\right)
$$

where $Z_{0}$ is the wave impedance in free space. Substituting Eq. (6) into Eq. (5), then equation (5) can be converted as: 


$$
\left.\begin{array}{c}
\mathbf{E}_{\mathrm{s}}(\mathbf{r}, \omega)=j k \frac{\exp (j k r)}{4 \pi r} \int_{S}\left\{\begin{array}{l}
\hat{\mathbf{n}} \times\left[\hat{\mathbf{k}}_{\mathrm{i}}\left(\mathbf{r}^{\prime}\right) \times \mathbf{E}\left(\mathbf{r}^{\prime}, \omega\right)\right] \\
+\hat{\mathbf{k}}_{\mathrm{s}} \times\left[\hat{\mathbf{n}} \times \mathbf{E}\left(\mathbf{r}^{\prime}, \omega\right)\right] \\
\left.-\left[\hat{\mathbf{n}} \cdot \mathbf{E}\left(\mathbf{r}^{\prime}, \omega\right)\right] \hat{\mathbf{k}}_{\mathrm{s}}\right)
\end{array}\right\} \exp \left(-j \mathbf{k}_{\mathrm{s}} \cdot \mathbf{r}^{\prime}\right) \mathrm{d} s^{\prime} \\
=j k \frac{\exp (j k r)}{4 \pi r} \int_{S}\left\{\begin{array}{l}
\hat{\mathbf{n}} \times\left[\hat{\mathbf{k}}_{\mathrm{i}}\left(\mathbf{r}^{\prime}\right) \times \mathbf{E}\left(\mathbf{r}^{\prime}, \omega\right)\right] \\
+\hat{\mathbf{n}}\left[\hat{\mathbf{k}}_{\mathrm{s}} \cdot \mathbf{E}\left(\mathbf{r}^{\prime}, \omega\right)\right] \\
-\hat{E}^{\prime}\left(\mathbf{r}^{\prime}, \omega\right)\left(\hat{\mathbf{k}}_{\mathrm{s}} \cdot \hat{\mathbf{n}}\right) \\
-\hat{\mathbf{k}}_{\mathrm{s}}\left[\hat{\mathbf{n}} \cdot \mathbf{E}\left(\mathbf{r}^{\prime}, \omega\right)\right]
\end{array}\right\} \exp \left(-j \mathbf{k}_{\mathrm{s}} \cdot \mathbf{r}^{\prime}\right) \mathrm{d} s^{\prime}
\end{array}\right\}
$$

The integral of the Eq. (7) with respect to the closed surface $S$ can be converted into the integral over the infinite plane $S^{\prime \prime}$. As shown in Fig. 2, since the plane $S^{\prime \prime}$ is perpendicular to the observation direction of the scattering fields, the relationship $\hat{\mathbf{k}}_{\mathrm{s}} \cdot \hat{\mathbf{n}}=1$ holds. Eliminating the similar items, equation (7) is finally reduced to:

$\mathbf{E}_{\mathrm{s}}(\mathbf{r}, \omega)=j k \frac{\exp (j k r)}{4 \pi r} \iint_{S^{\prime \prime}}\left\{\hat{\mathbf{n}} \times\left[\hat{\mathbf{k}}_{\mathrm{i}}\left(\mathbf{r}^{\prime}\right) \times \mathbf{E}\left(\mathbf{r}^{\prime}, \omega\right)\right]-\mathbf{E}\left(\mathbf{r}^{\prime}, \omega\right)\right\} \exp \left(-j k \hat{\mathbf{n}} \cdot \mathbf{r}^{\prime}\right) \mathrm{d} s^{\prime}$

This equation is equivalent to:

$\mathbf{E}_{\mathrm{s}}(\mathbf{r}, \omega)=j k \frac{\exp (j k r)}{4 \pi r} \iint_{S^{\prime \prime}}\left\{\hat{\mathbf{k}}_{\mathrm{s}} \times\left[\hat{\mathbf{k}}_{\mathrm{i}}\left(\mathbf{r}^{\prime}\right) \times \mathbf{E}\left(\mathbf{r}^{\prime}, \omega\right)\right]-\mathbf{E}\left(\mathbf{r}^{\prime}, \omega\right)\right\} \exp \left(-j \mathbf{k}_{\mathrm{s}} \cdot \mathbf{r}^{\prime}\right) \mathrm{d} s^{\prime}$

where $\hat{\mathbf{k}}_{\mathrm{i}}\left(\mathbf{r}^{\prime}\right)$ is the unit wave vector of the scattering waves arriving at the surface $S^{\prime \prime}$ from the scatterer, $\hat{\mathbf{k}}_{\mathrm{s}}$ represents the unit vector of the scattering observation direction, and $\mathbf{r}^{\prime}$ represents the location vector of any point on the integral plane $S^{\prime \prime}$.

Finally, according to the method given in Section 2.1, the integral of Eq. (8) over the infinite plane $S^{\prime \prime}$ can be transformed into the integral over the finite plane $S^{\prime}$, by changing the integral domain. This result shows that, as long as the electric fields at the finite plane $S^{\prime}$ is determined, the scattering far field can be obtained as well.

\subsubsection{Equation of the second kind}

Considering the far-field scattering, the scattering contribution mainly comes from plane $S^{\prime}$ of limited area. So the electric field on plane $S^{\prime}$ can be decomposed in both vertical and parallel directions. From a physical point of view, the field component vertical 
to the surface $S^{\prime}$ is approximately no contribution to the backscattering since $\hat{\mathbf{n}}=\hat{\mathbf{k}}_{\mathrm{s}}$. The main contribution comes from the field component parallel to the plane $S^{\prime}$. This physical characteristic means:

$$
\hat{\mathbf{n}} \cdot \mathbf{E}\left(\mathbf{r}^{\prime}, \omega\right) \approx 0
$$

Based on the integral domain transformation, substituting Eq. (9) into Eq. (1) yields:

$$
\mathbf{E}_{\mathbf{s}}(\mathbf{r}, \omega)=\iint_{S^{\prime}}\left\{j \omega \mu\left[\hat{\mathbf{n}} \times \mathbf{H}\left(\mathbf{r}^{\prime}, \omega\right)\right] G+\left[\hat{\mathbf{n}} \times \mathbf{E}\left(\mathbf{r}^{\prime}, \omega\right)\right] \times \nabla^{\prime} G\right\} \mathrm{d} s^{\prime}
$$

Then, according to Green's function, we can obtain:

$$
\begin{aligned}
\mathbf{E}_{\mathrm{s}}(\mathbf{r}, \omega) & =j \iint_{S^{\prime}} G\left\{\omega \mu\left[\hat{\mathbf{n}} \times \mathbf{H}\left(\mathbf{r}^{\prime}, \omega\right)\right]+\mathbf{k}_{\mathrm{s}} \times\left[\hat{\mathbf{n}} \times \mathbf{E}\left(\mathbf{r}^{\prime}, \omega\right)\right]\right\} \mathrm{d} s^{\prime} \\
& =j \frac{\exp (j k r)}{4 \pi r} \iint_{S^{\prime}}\left\{\begin{array}{l}
\omega \mu\left[\hat{\mathbf{n}} \times \mathbf{H}\left(\mathbf{r}^{\prime}, \omega\right)\right] \\
+\mathbf{k}_{\mathrm{s}} \times\left[\hat{\mathbf{n}} \times \mathbf{E}\left(\mathbf{r}^{\prime}, \omega\right)\right]
\end{array}\right\} \exp \left(-j \mathbf{k}_{\mathrm{s}} \cdot \mathbf{r}^{\prime}\right) \mathrm{d} s^{\prime}
\end{aligned}
$$

Substituting Eq. (6) into Eq. (11), the later equation can be simplified:

$$
\begin{aligned}
\mathbf{E}_{\mathrm{s}}(\mathbf{r}, \omega) & =j \frac{\exp (j k r)}{4 \pi r} \iint_{S^{\prime}}\left\{\begin{array}{l}
\omega \mu\left[\hat{\mathbf{k}}_{\mathrm{s}} \times \mathbf{H}\left(\mathbf{r}^{\prime}, \omega\right)\right] \\
+\mathbf{k}_{\mathrm{s}} \times\left[\hat{\mathbf{k}}_{\mathrm{s}} \times \mathbf{E}\left(\mathbf{r}^{\prime}, \omega\right)\right]
\end{array}\right\} \exp \left(-j \mathbf{k}_{\mathrm{s}} \cdot \mathbf{r}^{\prime}\right) \mathrm{d} s^{\prime} \\
& =j \frac{\exp (j k r)}{4 \pi r} \iint_{S^{\prime}}\left\{\begin{array}{l}
\omega \mu\left[\hat{\mathbf{k}}_{\mathrm{s}} \times \frac{\hat{\mathbf{k}}_{\mathrm{i}} \times \mathbf{E}\left(\mathbf{r}^{\prime}, \omega\right)}{Z_{0}}\right] \\
+\omega \sqrt{\varepsilon \mu} \hat{\mathbf{k}}_{\mathrm{s}} \times\left[\hat{\mathbf{k}}_{\mathrm{s}} \times \mathbf{E}\left(\mathbf{r}^{\prime}, \omega\right)\right]
\end{array}\right\} \exp \left(-j \mathbf{k}_{\mathrm{s}} \cdot \mathbf{r}^{\prime}\right) \mathrm{d} s^{\prime} \\
& =j k \frac{\exp (j k r)}{4 \pi r} \iint_{S^{\prime}}\left\{\begin{array}{l}
\hat{\mathbf{k}}_{\mathrm{s}} \times\left[\hat{\mathbf{k}}_{\mathrm{i}}\left(\mathbf{r}^{\prime}\right) \times \mathbf{E}\left(\mathbf{r}^{\prime}, \omega\right)\right] \\
+\hat{\mathbf{k}}_{\mathrm{s}} \times\left[\hat{\mathbf{k}}_{\mathrm{s}} \times \mathbf{E}\left(\mathbf{r}^{\prime}, \omega\right)\right]
\end{array}\right\} \exp \left(-j \mathbf{k}_{\mathrm{s}} \cdot \mathbf{r}^{\prime}\right) \mathrm{d} s^{\prime}
\end{aligned}
$$

The above derivation process is based on the relationship $\hat{\mathbf{n}}=\hat{\mathbf{k}}_{\mathrm{s}}$, which can be used again to rewritten Eq. (12a) as:

$\mathbf{E}_{\mathrm{s}}(\mathbf{r}, \omega)=j k \frac{\exp (j k r)}{4 \pi r} \iint_{S^{\prime}}\left\{\begin{array}{l}\hat{\mathbf{n}} \times\left[\hat{\mathbf{k}}_{\mathrm{i}}\left(\mathbf{r}^{\prime}\right) \times \mathbf{E}\left(\mathbf{r}^{\prime}, \omega\right)\right] \\ +\hat{\mathbf{n}} \times\left[\hat{\mathbf{n}} \times \mathbf{E}\left(\mathbf{r}^{\prime}, \omega\right)\right]\end{array}\right\} \exp \left(-j k \hat{\mathbf{n}} \cdot \mathbf{r}^{\prime}\right) \mathrm{d} s^{\prime}$ 
If the vertical field component on the plane $S^{\prime}$ is considered in the derivation of Eq. (12), equation (8) can also be derived. In addition, combined with the physical characteristics of backscattering, Eq. (8) can be derived directly from Eq. (12) as well, which is discussed next.

\subsection{Discussion on the consistency of the derived equations}

Taking Eq. (12a) as an example, it can be further changed as:

$$
\begin{aligned}
& \mathbf{E}_{\mathrm{s}}(\mathbf{r}, \omega)=j k \frac{\exp (j k r)}{4 \pi r} \iint_{S^{\prime}}\left\{\begin{array}{l}
\hat{\mathbf{k}}_{\mathrm{s}} \times\left[\hat{\mathbf{k}}_{\mathrm{i}}\left(\mathbf{r}^{\prime}\right) \times \mathbf{E}\left(\mathbf{r}^{\prime}, \omega\right)\right] \\
+\hat{\mathbf{k}}_{\mathrm{s}} \times\left[\hat{\mathbf{k}}_{\mathrm{s}} \times \mathbf{E}\left(\mathbf{r}^{\prime}, \omega\right)\right]
\end{array}\right\} \exp \left(-j \mathbf{k}_{\mathrm{s}} \cdot \mathbf{r}^{\prime}\right) \mathrm{d} s^{\prime} \\
& =j k \frac{\exp (j k r)}{4 \pi r} \iint_{S^{\prime}}\left\{\begin{array}{l}
\hat{\mathbf{k}}_{\mathrm{s}} \times\left[\hat{\mathbf{k}}_{\mathrm{i}}\left(\mathbf{r}^{\prime}\right) \times \mathbf{E}\left(\mathbf{r}^{\prime}, \omega\right)\right] \\
+\hat{\mathbf{k}}_{\mathrm{s}}\left[\hat{\mathbf{k}}_{\mathrm{s}} \cdot \mathbf{E}\left(\mathbf{r}^{\prime}, \omega\right)\right] \\
-\mathbf{E}\left(\mathbf{r}^{\prime}, \omega\right)\left(\hat{\mathbf{k}}_{\mathrm{s}} \cdot \hat{\mathbf{k}}_{\mathrm{s}}\right)
\end{array}\right\} \exp \left(-j \mathbf{k}_{\mathrm{s}} \cdot \mathbf{r}^{\prime}\right) \mathrm{d} s^{\prime} \\
& =j k \frac{\exp (j k r)}{4 \pi r} \iint_{S^{\prime}}\left\{\begin{array}{l}
\hat{\mathbf{k}}_{\mathrm{s}} \times\left[\hat{\mathbf{k}}_{\mathrm{i}}\left(\mathbf{r}^{\prime}\right) \times \mathbf{E}\left(\mathbf{r}^{\prime}, \omega\right)\right] \\
+\hat{\mathbf{k}}_{\mathrm{s}}\left[\hat{\mathbf{k}}_{\mathrm{s}} \cdot \mathbf{E}\left(\mathbf{r}^{\prime}, \omega\right)\right]-\mathbf{E}\left(\mathbf{r}^{\prime}, \omega\right)
\end{array}\right\} \exp \left(-j \mathbf{k}_{\mathrm{s}} \cdot \mathbf{r}^{\prime}\right) \mathrm{d} s^{\prime}
\end{aligned}
$$

To physically show the relationship between Eqs. (12) and (8), the electric fields on the surface $S^{\prime}$ are decomposed in the vertical and parallel directions:

$$
\mathbf{E}\left(\mathbf{r}^{\prime}, \omega\right)=\mathbf{E}_{\perp}\left(\mathbf{r}^{\prime}, \omega\right)+\mathbf{E}_{\|}\left(\mathbf{r}^{\prime}, \omega\right)
$$

Substituting it into Eq. (13) yields:

$$
\begin{aligned}
& \mathbf{E}_{\mathrm{s}}(\mathbf{r}, \omega)=j k \frac{\exp (j k r)}{4 \pi r} \iint_{S^{\prime}}\left\{\begin{array}{l}
\hat{\mathbf{k}}_{\mathrm{s}} \times\left[\hat{\mathbf{k}}_{\mathrm{i}}\left(\mathbf{r}^{\prime}\right) \times \mathbf{E}\left(\mathbf{r}^{\prime}, \omega\right)\right] \\
+\hat{\mathbf{k}}_{\mathrm{s}}\left[\hat{\mathbf{k}}_{\mathrm{s}} \cdot\left(\mathbf{E}_{\perp}\left(\mathbf{r}^{\prime}, \omega\right)+\mathbf{E}_{\|}\left(\mathbf{r}^{\prime}, \omega\right)\right)\right] \\
-\mathbf{E}\left(\mathbf{r}^{\prime}, \omega\right)
\end{array}\right\} \exp \left(-j \mathbf{k}_{\mathrm{s}} \cdot \mathbf{r}^{\prime}\right) \mathrm{d} s^{\prime} \\
& =j k \frac{\exp (j k r)}{4 \pi r} \iint_{S^{\prime}}\left\{\begin{array}{l}
\hat{\mathbf{k}}_{\mathrm{s}} \times\left[\hat{\mathbf{k}}_{\mathrm{i}}\left(\mathbf{r}^{\prime}\right) \times \mathbf{E}\left(\mathbf{r}^{\prime}, \omega\right)\right] \\
+\hat{\mathbf{k}}_{\mathrm{s}}\left[\hat{\mathbf{k}}_{\mathrm{s}} \cdot \mathbf{E}_{\perp}\left(\mathbf{r}^{\prime}, \omega\right)+\hat{\mathbf{k}}_{\mathrm{s}} \cdot \mathbf{E}_{\|}\left(\mathbf{r}^{\prime}, \omega\right)\right] \\
-\mathbf{E}\left(\mathbf{r}^{\prime}, \omega\right)
\end{array}\right\} \exp \left(-j \mathbf{k}_{\mathrm{s}} \cdot \mathbf{r}^{\prime}\right) \mathrm{d} s^{\prime}
\end{aligned}
$$


Obviously, the field component vertical to the plane $S^{\prime}$ has approximately no contribution to the backscattering; and considering the scattering contribution of each component, the following relationships result:

$$
\left\{\begin{array}{l}
\hat{\mathbf{n}}=\hat{\mathbf{k}}_{\mathrm{s}} \\
\mathbf{E}_{\|}\left(\mathbf{r}^{\prime}, \omega\right) \cdot \hat{\mathbf{k}}_{\mathrm{s}}=0 \\
\mathbf{E}_{\perp}\left(\mathbf{r}^{\prime}, \omega\right) \cdot \hat{\mathbf{k}}_{\mathrm{s}} \approx 0
\end{array}\right.
$$

Substituting Eq. (16) into Eq. (15), the following relationship can be obtained:

$$
\mathbf{E}_{\mathrm{s}}(\mathbf{r}, \omega)=j k \frac{\exp (j k r)}{4 \pi r} \iint_{S^{\prime}}\left\{\begin{array}{l}
\hat{\mathbf{k}}_{\mathrm{s}} \times\left[\hat{\mathbf{k}}_{\mathrm{i}}\left(\mathbf{r}^{\prime}\right) \times \mathbf{E}\left(\mathbf{r}^{\prime}, \omega\right)\right] \\
-\mathbf{E}\left(\mathbf{r}^{\prime}, \omega\right)
\end{array}\right\} \exp \left(-j \mathbf{k}_{\mathrm{s}} \cdot \mathbf{r}^{\prime}\right) \mathrm{d} s^{\prime}
$$

This relationship indicates the equivalence between Eqs. (8) and (12). The significance of this conclusion is that different approaches arriving at the same result can confirm the correctness of the result. In addition, further derivation based on Eq. (8) can be conducted:

$$
\begin{gathered}
\mathbf{E}_{\mathrm{s}}(\mathbf{r}, \omega)=j k \frac{\exp (j k r)}{4 \pi r} \iint_{S^{\prime}}\left\{\begin{array}{l}
\hat{\mathbf{k}}_{\mathrm{s}} \times\left[\hat{\mathbf{k}}_{\mathrm{i}}\left(\mathbf{r}^{\prime}\right) \times \mathbf{E}\left(\mathbf{r}^{\prime}, \omega\right)\right] \\
-\mathbf{E}\left(\mathbf{r}^{\prime}, \omega\right)
\end{array}\right\} \exp \left(-j \mathbf{k}_{\mathrm{s}} \cdot \mathbf{r}^{\prime}\right) \mathrm{d} s^{\prime} \\
=j k \frac{\exp (j k r)}{4 \pi r} \iint_{S^{\prime}}\left\{\begin{array}{l}
\hat{\mathbf{k}}_{\mathrm{i}}\left(\mathbf{r}^{\prime}\right) \times\left[\hat{\mathbf{k}}_{\mathrm{s}} \cdot \mathbf{E}\left(\mathbf{r}^{\prime}, \omega\right)\right] \\
-\mathbf{E}\left(\mathbf{r}^{\prime}, \omega\right)\left[\hat{\mathbf{k}}_{\mathrm{s}} \cdot \hat{\mathbf{k}}_{\mathrm{i}}\left(\mathbf{r}^{\prime}\right)\right] \\
-\mathbf{E}\left(\mathbf{r}^{\prime}, \omega\right)
\end{array}\right\} \exp \left(-j \mathbf{k}_{\mathrm{s}} \cdot \mathbf{r}^{\prime}\right) \mathrm{d} s^{\prime} \\
=j k \frac{\exp (j k r)}{4 \pi r} \iint_{S^{\prime}}\left\{\begin{array}{l}
\hat{\mathbf{k}}_{\mathrm{i}}\left(\mathbf{r}^{\prime}\right)\left[\hat{\mathbf{k}}_{\mathrm{s}} \cdot\left(\mathbf{E}_{\perp}\left(\mathbf{r}^{\prime}, \omega\right)+\mathbf{E}_{\|}\left(\mathbf{r}^{\prime}, \omega\right)\right)\right] \\
-\mathbf{E}\left(\mathbf{r}^{\prime}, \omega\right)\left[\hat{\mathbf{k}}_{\mathrm{s}} \cdot \hat{\mathbf{k}}_{\mathrm{i}}\left(\mathbf{r}^{\prime}\right)\right] \\
-\mathbf{E}\left(\mathbf{r}^{\prime}, \omega\right)
\end{array}\right\} \exp \left(-j \mathbf{k}_{\mathrm{s}} \cdot \mathbf{r}^{\prime}\right) \mathrm{d} s^{\prime}
\end{gathered}
$$

Substituting Eq. (16) into Eq. (17), the latter is simplified as:

$$
\mathbf{E}_{\mathrm{s}}(\mathbf{r}, \omega)=-j k \frac{\exp (j k r)}{4 \pi r} \iint_{S^{\prime}}\left\{\mathbf{E}\left(\mathbf{r}^{\prime}, \omega\right)\left[1+\hat{\mathbf{k}}_{\mathrm{s}} \cdot \hat{\mathbf{k}}_{\mathrm{i}}\left(\mathbf{r}^{\prime}\right)\right]\right\} \exp \left(-j \mathbf{k}_{\mathrm{s}} \cdot \mathbf{r}^{\prime}\right) \mathrm{d} s^{\prime}
$$


which is equivalent to:

$$
\mathbf{E}_{\mathrm{s}}(\mathbf{r}, \omega)=-j k \frac{\exp (j k r)}{4 \pi r} \iint_{S^{\prime}}\left\{\mathbf{E}\left(\mathbf{r}^{\prime}, \omega\right)\left[1+\hat{\mathbf{n}} \cdot \hat{\mathbf{k}}_{\mathrm{i}}\left(\mathbf{r}^{\prime}\right)\right]\right\} \exp \left(-j k \hat{\mathbf{n}} \cdot \mathbf{r}^{\prime}\right) \mathrm{d} s^{\prime}
$$

Compared with Eq. (1), the physical meaning of Eq. (18) is more obvious and intuitive. This equation shows that, if the incident direction $\left(\hat{\mathbf{k}}_{\mathbf{i}}\right)$ of the scattering wave is more consistent with the direction $\left(\hat{\mathbf{k}}_{\mathrm{s}}\right)$ of the scattering observation, the scattering effect at the observation point is stronger. Thus, according to Eq. (18), two extreme scattering cases result: when the direction of the scattering wave coincides with the direction of the scattering observation, namely $\hat{\mathbf{k}}_{\mathrm{s}} \cdot \hat{\mathbf{k}}_{\mathrm{i}}=1$, the scattering effect is the strongest case; conversely, if they are in the opposite direction, namely $\hat{\mathbf{k}}_{\mathrm{s}} \cdot \hat{\mathbf{k}}_{\mathrm{i}}=-1$, the scattering effect is zero. Obviously, the results reflect the physical meaning of the electromagnetic scattering.

\subsection{Discussion on the extrapolation in the time domain}

Using $\exp (-j \omega t)$ as the time harmonic factor, Eq. (18a) can be organized as follows:

$$
\begin{aligned}
\mathbf{E}_{\mathrm{s}}(\mathbf{r}, \omega) & =-j k \frac{\exp (j k r)}{4 \pi r} \iint_{S^{\prime}}\left\{\left[1+\hat{\mathbf{k}}_{\mathrm{s}} \cdot \hat{\mathbf{k}}_{\mathrm{i}}\left(\mathbf{r}^{\prime}\right)\right] \mathbf{E}\left(\mathbf{r}^{\prime}, \omega\right)\right\} \exp \left(-j \mathbf{k}_{\mathrm{s}} \cdot \mathbf{r}^{\prime}\right) \mathrm{d} s^{\prime} \\
& =\frac{1}{4 \pi c r} \iint_{S^{\prime}}\left\{(-j \omega) \mathbf{E}\left(\mathbf{r}^{\prime}, \omega\right) \exp \left[j\left(k r-\mathbf{k}_{\mathrm{s}} \cdot \mathbf{r}^{\prime}\right)\right]\left[1+\hat{\mathbf{k}}_{\mathrm{s}} \cdot \hat{\mathbf{k}}_{\mathrm{i}}\left(\mathbf{r}^{\prime}\right)\right]\right\} \mathrm{d} s^{\prime}
\end{aligned}
$$

The far-field scattering formula in the time domain can be obtained by performing the inverse Fourier transformation on Eq. (19):

$$
\begin{aligned}
\mathbf{E}_{\mathrm{s}}(\mathbf{r}, t) & =F\left\{\frac{1}{4 \pi c r} \iint_{S^{\prime}}\left\{(-j \omega) \mathbf{E}\left(\mathbf{r}^{\prime}, \omega\right) \exp \left[j\left(k r-\mathbf{k}_{\mathrm{s}} \cdot \mathbf{r}^{\prime}\right)\right]\left[1+\hat{\mathbf{k}}_{\mathrm{s}} \cdot \hat{\mathbf{k}}_{\mathrm{i}}\left(\mathbf{r}^{\prime}\right)\right]\right\} \mathrm{d} s^{\prime}\right\} \\
& =\frac{1}{4 \pi c r} \iint_{S^{\prime}}\left\{\frac{\partial \mathbf{E}\left(\mathbf{r}^{\prime}, t-\frac{1}{\omega}\left(k r-\mathbf{k}_{\mathrm{s}} \cdot \mathbf{r}^{\prime}\right)\right)}{\partial t}\left[1+\hat{\mathbf{k}}_{\mathrm{s}} \cdot \hat{\mathbf{k}}_{\mathrm{i}}\left(\mathbf{r}^{\prime}\right)\right]\right\} \mathrm{d} s^{\prime} \\
& =\frac{1}{4 \pi c r} \iint_{S^{\prime}}\left\{\frac{\partial \mathbf{E}\left(\mathbf{r}^{\prime}, t-\frac{1}{c}\left(r-\hat{\mathbf{k}}_{\mathrm{s}} \cdot \mathbf{r}^{\prime}\right)\right)}{\partial t}\left[1+\hat{\mathbf{k}}_{\mathrm{s}} \cdot \hat{\mathbf{k}}_{\mathrm{i}}\left(\mathbf{r}^{\prime}\right)\right]\right\} \mathrm{d} s^{\prime}
\end{aligned}
$$


According to the vector relationship $\hat{\mathbf{n}}=\hat{\mathbf{k}}_{\mathrm{s}}$ as shown in Fig. 2, another form of Eq. (20a) is available:

$\mathbf{E}_{\mathrm{s}}(\mathbf{r}, t)=\frac{1}{4 \pi c r} \iint_{S^{\prime}}\left\{\frac{\partial \mathbf{E}\left(\mathbf{r}^{\prime}, t-\frac{1}{c}\left(r-\hat{\mathbf{n}} \cdot \mathbf{r}^{\prime}\right)\right)}{\partial t}\left[1+\hat{\mathbf{n}} \cdot \hat{\mathbf{k}}_{\mathrm{i}}\left(\mathbf{r}^{\prime}\right)\right]\right\} \mathrm{d} s^{\prime}$

The derivation above shows that Eq. (1) can evolve into Eqs. (8), (12) and (18) three more concise forms, whose time-domain calculation can be easily obtained. One point worthy of note is that all derivations are based on the plane $S^{\prime \prime}$ (or $S^{\prime}$ ) vertical to the scattering direction, which is the precondition for deriving these three equations. Therefore, when the observation direction of the backscattering is changed, the orientation of the plane $S^{\prime \prime}$ should be changed as well. For example, when calculating the bistatic RCS, the plane $S^{\prime \prime}$ should be rotated as the observation point changes.

\section{Example}

A numerical example is given to further illustrate the validity of the derived result in this paper. As shown in Fig. 3a, an ideal metal plate whose width and length are both

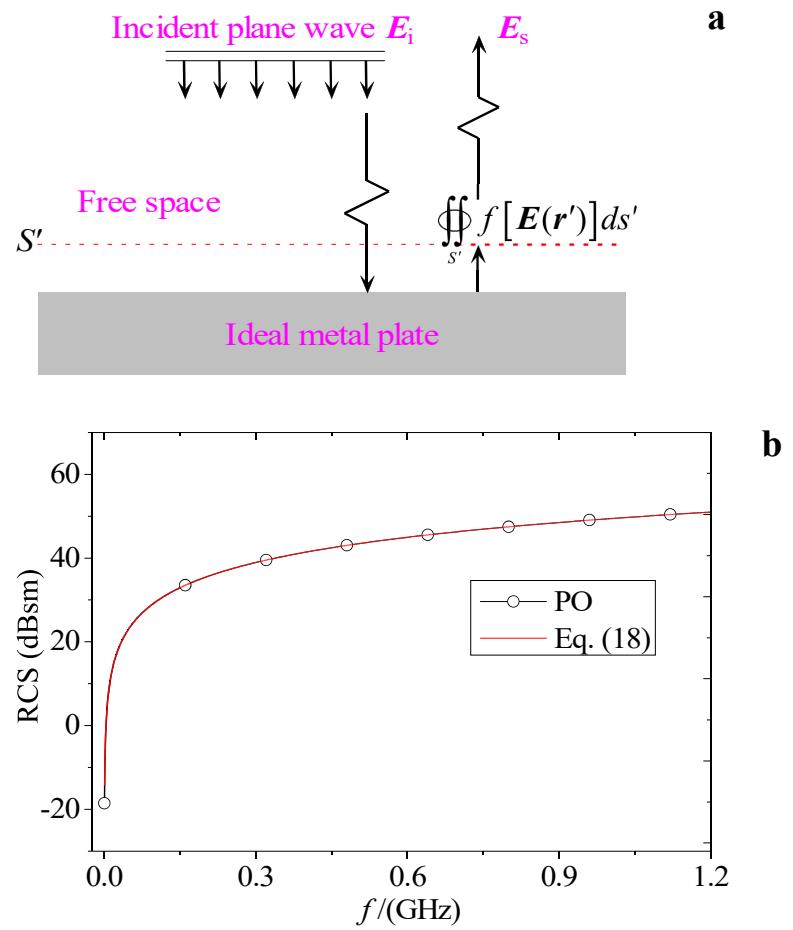

Fig. 3. Numerical example. (a) The schematic of the numerical model. (b) Characteristics of the backscattering. 
$5 \mathrm{~m}$ is selected as the scatterer. The Gaussian pulse incident wave illuminates the metal plate vertically. Figure $3 \mathbf{b}$ shows the monostatic RCS of the plate resulting from different methods, which confirms the correctness of the algorithm as well.

\section{Conclusion}

Based on the Stratton-Chu formula, the electric field extrapolation both in time domain and frequency domain is studied. The derivation process shows that the calculation accuracy of the formulas obtained is determined by the area of the integral plane, namely, the larger the area of the finite plane $S^{\prime}$, the more accurate the calculation. The consistency property of the results obtained from different derivation methods confirms the correctness of the results. In addition, numerical example verifies the correctness as well. Compared with the Stratton-Chu formula, the results in this paper are characterized by simple form and intuitive physical meaning; and it is easy to obtain the extrapolation formula in the time domain. More importantly, since the transformation method between the closed surface $S$, the infinite plane $S^{\prime \prime}$, and the finite plane $S^{\prime}$ is built, which is more convenient for the analysis of complex problems.

Acknowledgements - This project is financially supported by China Scholarship Council (Grant No. 202106675002), Open Foundation of Semiconductor Power Device Reliability Engineering Research Center of Ministry of Education, China (Grant No. ERCMEKFJJ2019-(05)), and Natural Science Foundation of Guizhou University, China (Grant No. (2019)62).

\section{References}

[1] Yee K.S., Ingham D., Shlager K., Time-domain extrapolation to the far field based on FDTD calculations, IEEE Transactions on Antennas and Propagation 39(3), 1991, pp. 410-413, DOI: $10.1109 /$ 8.76342.

[2] RAMAHI O.M., Near- and far-field calculations in FDTD simulations using Kirchhoff surface integral representation, IEEE Transactions on Antennas and Propagation 45(5), 1997, pp. 753-759, DOI: $10.1109 / 8.575616$.

[3] Marrocco G., Fari S., Bardati F., A hybrid FDTD-MoM procedure for the modeling of electromagnetic radiation from cavity-backed apertures, [In] IEEE Antennas and Propagation Society International Symposium. 2001 Digest. Held in conjunction with: USNC/URSI National Radio Science Meeting (Cat. No.01CH37229), Vol. 4, 2001, pp. 302-305, DOI: 10.1109/APS.2001.959458.

[4] Sнum S.M., Luk K.M., An efficient FDTD near-to-far-field transformation for radiation pattern calculation, Microwave and Optical Technology Letters 20(2), 1999, pp. 129-131, DOI: 10.1002/(SICI) 1098-2760(19990120)20:2<129::AID-MOP14>3.0.CO;2-C.

[5] Koh J., Lee W., Sarkar T.K., Salazar-Palma M., Calculation of far-field radiation pattern using nonuniformly spaced antennas by a least square method, IEEE Transactions on Antennas and Propagation 62(4), 2014, pp. 1572-1578, DOI: 10.1109/TAP.2013.2293146.

[6] Stratton J.A., Chu L.J., Diffraction theory of electromagnetic waves, Physical Review 56(1), 1939, pp. 99-107, DOI: 10.1103/PhysRev.56.99.

[7] Stratton J.A., Electromagnetic Theory, McGraw-Hill, New York, 1941.

[8] MAger R., BLEISTEIn N., An examination of the limited aperture problem of physical optics inverse scattering, IEEE Transactions on Antennas and Propagation 26(5), 1978, pp. 695-699, DOI: 10.1109/ TAP.1978.1141914. 
[9] Sarabandi K., Chiu T.C., Optimum corner reflectors for calibration of imaging radars, IEEE Transactions on Antennas and Propagation 44(10), 1996, pp. 1348-1361, DOI: 10.1109/8.537329.

[10] Vico-Bondia F., Ferrando-Bataller M., Valero-Nogueira A., A new fast physical optics for smooth surfaces by means of a numerical theory of diffraction, IEEE Transactions on Antennas and Propagation 58(3), 2010, pp. 773-789, DOI: 10.1109/TAP.2009.2039308.

Received May 1, 2020 